\title{
TIME TO EXTINCTION OF BIRD POPULATIONS
}

\author{
Bernt-Erik S Æther, ${ }^{1,15}$ Steinar Engen, ${ }^{2}$ Anders Pape Møller, ${ }^{3}$ Marcel E. Visser,${ }^{4}$ Erik Matthysen, ${ }^{5}$ \\ Wolfgang Fiedler,${ }^{6}$ Marcel M. Lambrechts,${ }^{7}$ Peter H. Becker,${ }^{8}$ Jon E. Brommer,${ }^{9}$ \\ Janis Dickinson, ${ }^{10}$ Chris du Feu, ${ }^{4}$ Frederick R. Gehlbach, ${ }^{11}$ Juha Merilä,,${ }^{9}$ Wallace Rendell,${ }^{12}$ \\ Raleigh J. Robertson, ${ }^{13}$ DAVID ThOMson, ${ }^{4}$ AND JÁNOS TÖRÖK ${ }^{14}$ \\ ${ }^{1}$ Department of Biology, Norwegian University of Science and Technology, N-7491 Trondheim, Norway \\ ${ }^{2}$ Department of Mathematical Sciences, Norwegian University of Science and Technology, N-7491 Trondheim, Norway \\ ${ }^{3}$ Laboratoire de Parasitologie Evolutive, CNRS UMR 7103, Université Pierre et Marie Curie, Bâtiment A, 7ème étage, \\ 7 quai St. Bernard, Case 237, FR-75252 Paris Cedex 05, France \\ ${ }^{4}$ Netherlands Institute of Ecology, P.O. Box 40, 6666 ZG Heteren, The Netherlands \\ ${ }_{5}^{5}$ Laboratory of Animal Ecology, Department of Biology, University of Antwerp, B-2610 Antwerp, Belgium \\ ${ }^{6}$ Max Planck Research Center for Ornithology, Vogelwarte Radolfzell, Schlossallee 2, D-78315 Radolfzell, Germany \\ ${ }^{7}$ Laboratoire Centre d'Ecologie Fonctionnelle et Evolutive, Centre National de la Recherche Scientifique, \\ 1919 Route de Mende, F-34293 Montpellier Cedex 5, France \\ ${ }^{8}$ Institut für Vogelforschung, Vogelwarte Helgoland, An der Vogelwarte 21, D-26386 Wilhelmshaven, Germany \\ ${ }^{9}$ Department of Bio- and Environmental Sciences, P.O. Box 65 (Biocenter 3, Viikinkaari 1), FIN-00014 \\ University of Helsinki, Finland \\ ${ }^{10}$ Museum of Vertebrate Zoology, Hastings Natural History Reservation, 38601 E. Carmel Valley Road, \\ Carmel Valley, California 93924 USA \\ ${ }^{11}$ Department of Biology, Baylor University, Waco, Texas, 76798-7388 USA \\ ${ }^{12} 189$ Springhead Garden \#10, Richmond Hill, Ontario L4C 5C7, Canada \\ ${ }^{13}$ Department of Biology, Queen's University, Kingston, Ontario, K7L 3N6, Canada \\ ${ }^{14}$ Behavioural Ecology Group, Department of Systematics, Zoology \& Ecology, Eötvös University, H-1117, \\ Budapest, Pázmány P. sétány 1/C, Hungary
}

Abstract. The risk of extinction of populations has not previously been empirically related to parameters characterizing their population dynamics. To analyze this relationship, we simulated how the distribution of population dynamical characters changed as a function of time, in both the remaining and the extinct populations. We found for a set of 38 bird populations that environmental stochasticity had the most immediate effect on the risk of extinction, whereas the long-term persistence of the population was most strongly affected by the specific population growth rate. This illustrates the importance of including information on temporal trends in population size when assessing the viability of a population. We used these relationships to examine whether time to extinction can be predicted from interspecific life history variation. Two alternative hypotheses were examined. (1) Time to extinction should decrease with increasing clutch size or decreasing survival rate because of the larger stochastic components in the population dynamics of such species. (2) Time to extinction should increase with decreasing clutch size or longer life expectancy if extinction rates are most strongly influenced by variation in the specific population growth rate. In the present data set, time to extinction increased with decreasing clutch size because of larger stochastic influences on the population dynamics of species with large clutch sizes located toward the fast end of the "slow-fast continuum" of life history variation. This demonstrates that interspecific variation in extinction risk can be predicted from knowledge of general life history characteristics. Such information can therefore be useful for assessing minimum sizes of viable populations of birds.

Key words: birds; clutch size; comparative analyses; demography; environmental stochasticity; life history variation; PVA; stochastic population dynamics; population growth rate; time to extinction; viable population size.

\section{INTRODUCTION}

Extinction is one of the most important ecological processes affecting biological diversity. With various kinds of human activity causing rapid loss of species (May et al. 1995, Pimm et al. 1995), reducing the im-

Manuscript received 25 May 2004; accepted 15 July 2004; final version received 12 August 2004. Corresponding Editor: B. J. Danielson.

${ }^{15}$ E-mail: Bernt-Erik.Sather@bio.ntnu.no pact of factors affecting the extinction risk has been the major focus for many management plans for biodiversity conservation. This requires general insight as to how different factors are likely to influence time to extinction (Purvis et al. 2000a). It is surprising that few general hypotheses have been developed to explain variation in extinction risk, even in well-known taxa such as birds.

Five different approaches have been used in comparative studies of extinction risks. (1) A few studies 
(Pimm et al. 1988, Ferraz et al. 2003, Schoener et al. 2003) provide estimates of loss of species in a specific area over time. In some of those cases, species-specific risks of extinction have been related to ecological variables (Pimm et al. 1988, 1993) or life history traits (Pimm et al. 1993, Schoener et al. 2003). Obtaining unbiased estimates of extinction risk in such studies is difficult because of problems of sampling individuals of rare species and distinguishing between transient and stationary individuals. (2) The number of lost species may also be derived from species-area relationships (May et al. 1995) in which the number of species increases with area in a curvilinear way (Rosenzweig 1995). Such analyses have been used to predict the number of species lost as function of a reduction of the area of suitable habitat, and have been used especially to assess the consequences of deforestation (Pimm and Askins 1995), notably in tropical areas (Brooks et al. 1997, 1999). Unfortunately, these analyses are sensitive to uncertainties in the estimates of the parameters specifying the species-area relationship (Simberloff 1992). (3) The World Conservation Union (IUCN 2001) has developed a set of criteria for classification of populations and species into different categories of vulnerability. Analyses including several taxa (Owens and Bennett 2000, Purvis et al. 2000b, Bennett and Owens 2002, Fisher et al. 2003, Jones et al. 2003) have shown that species that are threatened by extinction are not randomly distributed within the phylogeny. A problem with these analyses is the use of qualitative criteria for assigning many species to different risk categories that often do not include any information on population trends. (4) Time to extinction may be assessed from patterns in time series of population fluctuations (Inchausti and Halley 2003). Such analyses have been used to characterize the vulnerability of a large number of populations (Fagan et al. 2001), ignoring that population variability is a function of many population parameters, each of which may affect the time to extinction differentially. Furthermore, many population estimates are strongly biased, making future population projections uncertain (De Valpine and Hastings 2002, Ellner et al. 2002). (5) The aim of population viability analysis is to predict risk of extinction, given a set of preconditions (Morris and Doak 2002). Quantitative estimates of population viability are now available for a large number of populations (Dennis et al. 1991, Burgman et al. 1993, Beissinger and Westphal 1998, Groom and Pascual 1998, Beissinger and McCullough 2002). Recently these analyses have been severely criticized because of large uncertainties in parameter estimates (Ludwig 1996a, 1999, Fieberg and Ellner 2000, Ellner et al. 2002) and because important parameters such as demographic stochasticity often are not properly included in the analyses (Sæther et al. 2000).

Here we will extend this latter approach and present quantitative analyses of variation in the expected time to extinction based on statistical estimates of parameters affecting the probability of extinction. Theoretical analyses have shown that the expected time to extinction of a population should increase with population size (Leigh 1981, Lande 1993). However, this general relationship has been difficult to support empirically (Schoener and Spiller 1992, Belovsky et al. 1999, Schoener et al. 2003), not the least because the expected time to extinction is influenced not only by population size, but also by parameters characterizing the expected dynamics of the populations, such as the specific growth rate at low densities and the form and strength of the density dependence (Sæther and Engen 2003, Henle et al. 2004). The expected time to extinction is also strongly affected by stochastic factors, caused by either demographic or environmental stochasticity (Lande 1998, Lande et al. 2003, Drake and Lodge 2004). Demographic stochasticity is random variation among individuals in fitness contributions (Engen et al. 1998), whereas environmental stochasticity is random variation affecting the whole or parts of the population similarly (Lande et al. 2003). Ignoring these stochastic effects may lead to serious overestimates of time to extinction (Lande 1998, Sæther and Engen 2003, Lande et al. 2003). For instance, Lande (1993) used a simple model (exponential growth up to a ceiling at the carrying capacity $K$ ) to show that the expected time to extinction with only demographic stochasticity present increases almost exponentially with $K$, whereas the scaling under the influence of environmental stochasticity is a power function of $K$. Including demographic stochasticity is especially important when assessing the extinction risk at small population sizes, whereas environmental stochasticity affects the expected lifetime of large populations as well (Lande et al. 2003). The relative contribution of the different parameters to variation in time to extinction, however, is poorly understood (Caughley 1994).

Interestingly, variation in parameters characterizing avian population dynamics is correlated with differences in life history (Sæther and Engen 2002, Sæther et al. 2002b). For instance, the specific growth rate is generally smaller in long-lived species whereas the stochastic components of the population dynamics increase among species with clutch size (Sæther et al. $2002 b$; Sæther et al., in press). This suggests that characteristics of the extinction process should also be related to life history variation. Such a relationship would be of great importance for development of management plans of threatened or vulnerable species with poorly known ecology because it suggests that minimum size of viable populations is a function of more easily collected life history data. Two general relationships between time to extinction and life history characteristics are possible (Pimm 1991), comparing populations of initially the same size and equal carrying capacities. If extinction occurs mainly due to stochastic effects, time to extinction should decrease with the average indi- 
vidual longevity. A short life expectancy is generally found among species located toward the fast end of the slow-fast continuum of avian life history covariation (Sæther and Bakke 2000, Bennett and Owens 2002). This occurs because of larger stochastic components in the population dynamics of such species. In contrast, higher extinction rates are expected in long-lived species if interspecific variation in time to extinction is most strongly influenced by variation in the specific population growth rate $r_{1}$ (Sæther et al. $2002 b$, Bennett and Owens 2002), which strongly influences the deterministic components of population dynamics.

The presence of any such relationships will facilitate parameterization of models used in population viability analysis, which is one of the cornerstones in conservation biology because it aims to provide a quantitative estimate of the cumulative probability of extinction within a specified time period (Mace and Lande 1991, Beissinger and McCullough 2002). Our purpose is to examine, by extensive use of diffusion approximations (Lande et al. 2003), how differences in various population parameters affect variation among stationary bird populations in the expected time to extinction.

\section{Population Model}

We use the logistic model of density regulation (May 1981) that has been found to describe the density dependence in bird populations quite well (Sæther and Engen 2002):

$$
\begin{aligned}
\mathrm{E}(\Delta N \mid N) & =r_{1} N\left(1-\frac{N-1}{K-1}\right) \\
\operatorname{var}(\Delta N \mid N) & =\sigma_{\mathrm{e}}^{2} N^{2}+\sigma_{\mathrm{d}}^{2} N .
\end{aligned}
$$

Here, $\mathrm{E}$ is the expectation, $N$ is the population size, $K$ is the carrying capacity, $r_{1}$ is the specific growth rate at $N=1$, and $\sigma_{\mathrm{d}}^{2}$ and $\sigma_{\mathrm{e}}^{2}$ are the demographic and environmental variance, respectively.

Expected time to extinction was calculated using diffusion approximations (Karlin and Taylor 1981). The diffusion approximation for the logistic model (for $x$ $=\ln N$ corresponding to Eqs. 1) has infinitesimal mean

$$
\mu(x)=r\left(1-\frac{e^{x}-1}{K-1}\right)-\frac{1}{2} \sigma_{\mathrm{e}}^{2}-\frac{1}{2} \sigma_{\mathrm{d}}^{2} e^{-x}
$$

and variance

$$
v(x)=\sigma_{\mathrm{e}}^{2}+\sigma_{\mathrm{d}}^{2} e^{-x} .
$$

The Green function $Y$ (Karlin and Taylor 1981) for the process is defined as

$$
Y\left(x, x_{0}\right)= \begin{cases}\frac{S(x)}{S(x) v(x)} & \text { for } x \leq x_{0} \\ \frac{S\left(x_{0}\right)}{s(x) v(x)} & \text { for } x>x_{0}\end{cases}
$$

where

$$
\begin{aligned}
& s(x)=\exp \left[-2 \int^{x}(\mu(u) / v(u)) d u\right] \\
& S(x)=\int^{x} s(u) d u
\end{aligned}
$$

and the lower integration boundary can be chosen arbitrarily (Karlin and Taylor 1981, Lande et al. 2003). The expected time to extinction at $X=0$ is then

$$
T=\int_{0}^{\infty} \Upsilon\left(x, x_{0}\right) d x .
$$

The use of diffusion approximations in studies of population processes has been questioned because it assumes that the population generally changes in small steps (Ludwig 1996b). However, extensive simulation studies have shown that diffusion formulas often give a remarkably accurate description of discrete population processes (Engen et al. 2003).

\section{Methods \\ Population data}

In our analyses we included long-term ( $\geq 10$ years) time series of fluctuations of populations in which population estimates were based on direct nest counts or where a large proportion of the individuals could be recognized. In this way we hoped to reduce the effects of sample errors in population estimates that can seriously bias estimates of population parameters (Lande et al. 2003). In all populations included in the present analyses, data on the return from one year to another of breeding females, as well as their offspring, were also available, and the recapture rates were high. To reduce the effects of autocorrelation in the time series due to age-structure effects (Lande et al. 2002), we only included species in which the modal age of maturity among the females was 3 years old or less. In this way, we obtained data from 38 populations in 18 species (Appendix).

\section{Life history variables}

Modal values of clutch size and age at maturity $\alpha$ were used as estimates of these life history variables. The mean recapture rate of individually recognizable breeding females was used as an estimate of adult survival rate $s$. The generation time was estimated (Lande et al. 2003) as $G=\alpha+s /(1-s)$, where $\alpha$ is age at first reproduction, assuming a stable age structure and no age specificity in life history traits after sexual maturity.

\section{Estimation of population parameters}

The demographic variance $\sigma_{\mathrm{d}}^{2}$ is a measure of random variation among females in their annual fitness (Engen et al. 1998). The annual fitness of a female $i$ in year $t$, denoted $R_{i}$, is the number of female offspring born during the year that survive for at least one year plus 
1 if the female survives to the next year. The demographic variance was estimated for species that were sexually mature as 1-year-olds (Sæther et al. 1998) as the weighted mean across years of $\sigma_{\mathrm{d}}^{2}(t)=\mathrm{E}(1 /[a-$ 1]) $\Sigma\left(R_{i}-\bar{R}\right)^{2}$, where $\bar{R}$ is the mean contribution of the individuals and $a$ is the number of females monitored in year $t$. For species that matured at later ages we used the following (S. Engen, R. Lande, and B.-E. Sæther, unpublished manuscript):

$$
\begin{aligned}
\hat{\sigma}_{\mathrm{d}}^{2}=\sum_{i=0}^{k} \frac{1}{U_{i}} & {\left[\left(\frac{\partial r}{\partial F_{i}}\right)^{2} \hat{\sigma}_{F_{i}}^{2}+\left(\frac{\partial r}{\partial S_{i}}\right)^{2} \hat{\sigma}_{S_{i}}^{2}\right.} \\
& \left.+2 \frac{\partial r}{\partial F_{i}} \frac{\partial r}{\partial S_{i}} \widehat{\operatorname{cov}}\left(F_{i}, S_{i}\right)\right] .
\end{aligned}
$$

Here $r=\ln \Gamma$, where $\Gamma$ is the dominant eigenvalue of the mean Leslie matrix; $\hat{\sigma}_{F_{i}}^{2}$ and $\hat{\sigma}_{S_{i}}^{2}$ are the unbiased estimators for the components of $\sigma_{\mathrm{d}}^{2}$ from fecundity $F_{i}$ and survival $S_{i}$ in age class $i$, respectively; $\widehat{\operatorname{cov}}\left(F_{i}, S_{i}\right)$ is the estimate of the covariance between $F_{i}$ and $S_{i}$ in age class $i$; and $\left(U_{0}, U_{1}, \ldots, U_{k}\right)$ is the stable age distribution for the deterministic matrix model.

The parameters in Eqs. 1 were estimated by maximum likelihood using the full likelihood function for the process obtained by assuming $\sigma_{\mathrm{d}}^{2}$ to be known and that change in (log-) population size $\Delta X$ conditioned on the logarithm of the population size $X$ is normally distributed (Sæther et al. 2000, 2002a).

\section{Conditional composition of parameter values}

Many populations will fluctuate around their carrying capacity for a long period of time before they eventually go extinct (Sæther and Engen 2003). Time to extinction is then approximately exponentially distributed with mean value $T$ given by Eq. 3 . The probability that the population is not extinct at time $t$ is therefore approximately $p=e^{-t / T}$. In our analysis we consider $n$ populations, subject to the logistic model of density regulation (Eqs. 1), at an initial size at $X_{0}=$ $\ln K$, where $K$ is the estimated carrying capacity of the population. Write $p_{i}(t)=e^{-t / T_{i}}$ for the probability that population $i$ has not gone extinct at time $t$, where $T_{i}$ is the expected time to extinction of population $i$. Let $\beta_{i}$ $=\left(\beta_{i 1}, \beta_{i 2}, \ldots, \beta_{i 4}\right)$ be the parameter values $\left(r_{1}, K\right.$, $\left.\sigma_{\mathrm{e}}^{2}, \sigma_{\mathrm{d}}^{2}\right)$ for population $i$ and let $B_{0}=\left(B_{01}, B_{02}, \ldots, B_{04}\right)$ be the value of $\beta_{i}$ for a randomly selected population at time $t=0$. Hence, initially the distribution of the parameters across populations is given by, $P\left(B=b_{i}\right)$ $=1 / n, i=1,2, \ldots, n$. At time $t>0$ populations may have gone extinct. The distribution of $B_{t}$, defined as the distribution of parameters of a randomly selected population at time $t$, conditioned on this population not being extinct, is

$$
P\left(B_{t}=\beta_{i}\right)=\frac{p_{i}(t)}{\sum_{j=1}^{n} p_{j}(t)}=q_{i}(t) .
$$

This defines the expected multivariate distribution of the parameters across non-extinct populations at time $t$. From Eq. 4 we obtain

$$
\begin{aligned}
\mathrm{E} B_{t j} & =\sum \beta_{i j} q_{i}(t) \\
\operatorname{var}\left(B_{t j}\right) & =\sum \beta_{i j}^{2} q_{i}(t)-\left(\mathrm{E} B_{t j}\right)^{2} \\
\operatorname{cov}\left(B_{t j}, B_{t k}\right) & =\sum \beta_{i j} \beta_{i k} q_{i}(t)-\mathrm{E} B_{t j} \mathrm{E} B_{t k}
\end{aligned}
$$

which also defines the correlations

$$
\operatorname{corr}\left(B_{t j}, B_{t k}\right)=\frac{\operatorname{cov}\left(B_{t j}, B_{t k}\right)}{\sqrt{\operatorname{var}\left(B_{t j}\right) \operatorname{var}\left(B_{t k}\right)}} .
$$

This enables us to study how population extinctions through time are expected to change the characteristics of the multivariate distribution across populations of the parameter estimates.

\section{Phylogenetic analysis}

Phylogenetic analysis was performed using the $\lambda$ statistic, introduced by Pagel (1999) and Freckleton et al. (2002), which measures how closely the variation in traits across species accords with the predictions of a Brownian model for trait evolution. $\lambda$ varies between 0 (traits vary independently of phylogeny) and 1 (traits are distributed according to the Brownian model). $\lambda$ is estimated by maximum likelihood, and likelihood ratio tests may be used to determine whether maximum likelihood values differ significantly from 0 or 1 (Freckleton et al. 2002). The maximum likelihood value of $\lambda$ may be used to correct analyses for phylogenetic dependence, in particular to adjust data for the observed level of phylogenetic dependence in the data set. In the analysis that we present, correlations are reported at the maximum likelihood value of $\lambda$.

\section{RESUlts}

Simulating the time to extinction typically showed a negative exponential distribution. As shown in Fig. 1 , large differences could be found in the distribution of time to extinction, even between closely related species. We also noted that there was an initial period in which no extinctions occurred.

We utilized the exponential distribution of time to extinction (Fig. 1) to examine how the distribution of parameter values among populations changes through time due to extinctions (Eqs. 4-5). In the beginning, small changes were recorded in the composition of the populations (Fig. 2a). After this initial period, the population losses were approximately constant per time unit. This loss of populations over time strongly affected the distribution of the parameters in the remaining populations (Fig. 2b). First, a decrease occurred in the environmental variance, indicating that populations with a high environmental stochasticity were likely to go extinct first. Then an increase occurred throughout the whole period in the two parameters $r$ and $K$ that specify the deterministic dynamics, but more strongly in $r_{1}$ than in $K$. The demographic 


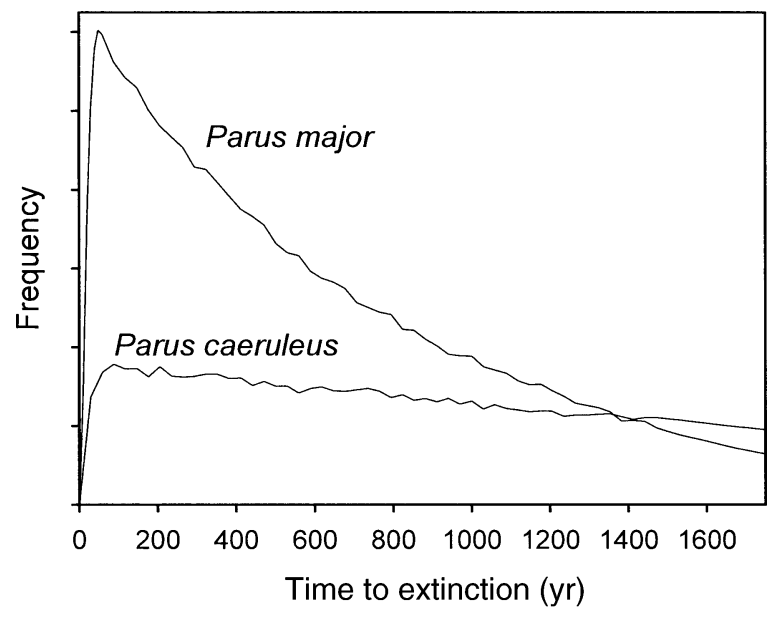

FIG. 1. Simulation of the distribution of time to extinction in a population of the Great Tit Parus major population at the island of Vlieland, The Netherlands (initial population size $N_{0}=K=114$ ), and in a population of Blue Tit Parus caeruleus at Corsica, France $\left(N_{0}=K=54\right)$. The number of simulations was 1000000 .

stochasticity seemed to influence the extinction process differentially because a reduction in the demographic variance of the remaining populations started to occur after a relatively long period of time (Fig. 2b). This indicates that the extinction process of bird populations can be characterized by an initial period in which populations are lost mainly due to the effects of environmental stochasticity, but in which long-term persistence is also determined by the deterministic components $\left(r_{1}\right.$ and $\left.K\right)$ of the population dynamics. As a consequence, the correlation coefficient (Eq. $5 \mathrm{~d}$ ) between $r_{1}$ and the stochastic component of the dynamics of the surviving populations increased through time (the correlation coefficient increased from 0.13 to 0.26 and from 0.23 to 0.33 with $\sigma_{\mathrm{e}}^{2}$ and $\sigma_{\mathrm{d}}^{2}$, respectively). This demonstrates an interaction through time between the deterministic and stochastic parts of the population dynamics. In contrast, the correlation between $r$ and $K$ increased only from 0.25 to 0.29 .

We have shown that deterministic components and the stochastic factors affect the long-term persistence of the populations differentially (Fig. 2b). Thus, in order to examine the hypotheses on the extinction risk and general life history characters, we related the expected time to extinction with life history characteristics for each species. To do so, we computed the mean equilibrium population size required to produce an expected time to extinction $T$ of 1000 years $\left(\log _{10} K_{T=1000}\right)$, i.e., how large the carrying capacity of a population need to be for the population not to go extinct during a period of 1000 years. These estimates were then related to the mean species-specific values of the population parameters and two major life history variables: clutch size and adult survival rate. The size of $\log _{10} K_{T=1000}$ increased with $\sigma_{\mathrm{e}}^{2}$ (correlation coefficient $=$
0.54, $P=0.02, n=18$; phylogenetic correlation $=$ $0.51, P<0.025(\lambda=0.042))$, but was not significantly related to any other population dynamic parameter. When we accounted for the positive correlation between $r_{1}$ and $\sigma_{\mathrm{e}}^{2}$ (correlation coefficient $=0.44, P=$ 0.07 ) in a multiple regression analysis, variation in both $\sigma_{\mathrm{e}}^{2}$ (standardized regression coefficient $=0.80, P<$ 0.001 ) and $r_{1}$ (standardized regression coefficient $=$ $-0.59, P=0.007)$ explained a significant proportion of the interspecific differences in $\log _{10} K_{T=1000}$. As expected from the life history correlates of population dynamics in birds (Sæther and Engen 2002, Sæther et al. 2002b), size of $\log _{10} K_{T=1000}$ increased with clutch size (Fig. 3; correlation coefficient $=0.61, P=0.007$, $n=18$; phylogenetic correlation $=0.63, P<0.005$ $(\lambda=0.182))$, but was only weakly correlated with adult survival rate (correlation coefficient $=-0.44, P=$ $0.07, n=18$ ) and generation time $G$ (correlation coefficient $=-0.32, P>0.19, n=18$ ). However, after accounting for phylogeny, $\log _{10} K_{T=1000}$ decreased sig-
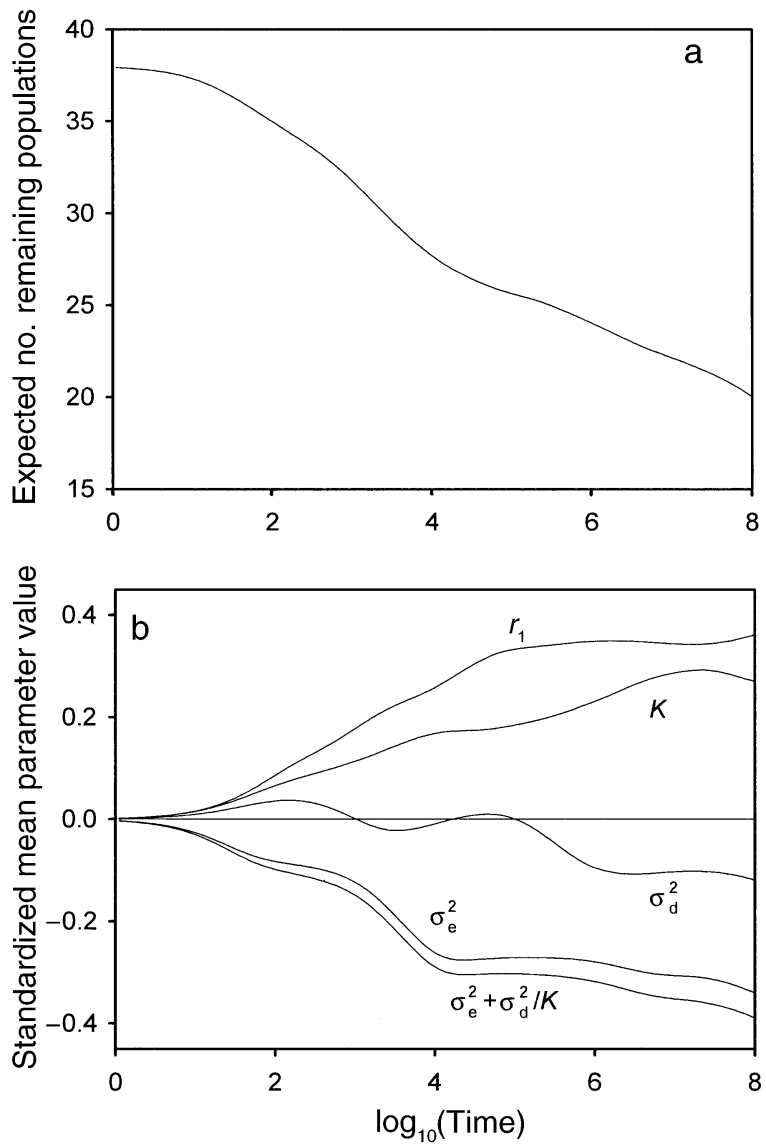

FIG. 2. Temporal changes in (a) the expected number of populations remaining and (b) the expected value of parameters in the remaining populations standardized according to the initial distributions of parameters across populations. Here $K$ is the carrying capacity, $r_{1}$ is the specific growth rate at $N$ $=1, \sigma_{\mathrm{d}}^{2}$ is the demographic variance, and $\sigma_{\mathrm{e}}^{2}$ is the environmental variance. 


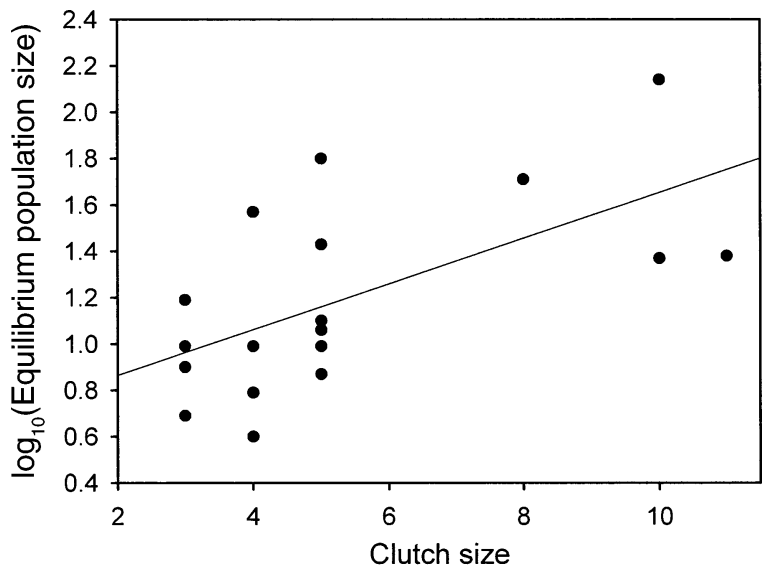

FIG. 3. The mean of the $\left(\log _{10}\right)$ equilibrium population size needed to produce an expected time to extinction $T$ equal to 1000 years $\left(\log _{10} K_{T=1000}\right)$ for each species in relation to interspecific differences in clutch size. The regression line (solid line) is $y=1.443 x+0.194 ; P=0.005$.

nificantly with adult survival rate (phylogenetic correlation $=-0.46, P<0.05(\lambda=0))$. This indicates that the population size necessary for keeping the expected time to extinction at $T=t$ increases with the position of the species toward the fast end of the slowfast continuum of avian life history covariation (Sæther and Bakke 2000, Bennett and Owens 2002). Although the index of phylogenetic dependence $\lambda$ indicated that both clutch size and adult survival were strongly linked to phylogeny ( $\lambda=1$ in both cases; likelihood ratio tests against $\lambda=0$ : clutch size, $\chi^{2}=8.692, P=0.003$; adult survival, $\left.\chi^{2}=11.388, P<0.001\right), \log _{10} K_{T=1000}$ showed no strong evidence of phylogenetic dependence $\left(\lambda=0.382\right.$, likelihood ratio test against $\lambda=0: \chi^{2}=$ $2.134, P=0.144)$. This suggests that both species- and population-specific factors can strongly influence the population size necessary for persistence.

\section{DISCUSSION}

Our results show that estimation of expected times to extinction of bird populations requires knowledge about the deterministic component as well as the stochastic effects on the population dynamics (Fig. 2b). However, we have shown on top of the relationship between expected time to extinction and population size that there is a clear effect of species-specific differences in extinction risk (Fig. 3). This occurs because interspecific stochastic influences on avian population dynamics are in part related to variation in life history traits; i.e., environmental stochasticity (Sæther and Engen 2002, Sæther et al. 2002b) and demographic stochasticity (Sæther et al., in press) increase with clutch size. Because stochastic effects reduce the long-run growth rate (Eq. 1a), a larger specific growth rate $r$ is necessary for population persistence in highly fecund than in long-lived species. However, more precise predictions of extinction risks can only be obtained when data about local population parameters are available, because there is large interpopulation variation in expected time to extinction (Fig. 1, Appendix).

An examination of the estimated expected times to extinction shows extremely long times to extinction (Appendix). This occurs because only populations with stationary fluctuations around $K$ are included in our analyses. This implies that we assume that current environmental conditions also will prevail in the future, which, of course, is not necessarily true. For instance, a reduction of $K$ or the specific growth rate, e.g., because of habitat loss or environmental deterioration, will strongly reduce the expected time to extinction (Lande et al. 2003, Sæther and Engen 2003). Furthermore, long-term climatic trends (Houghton et al. 2001) as well as temporal changes in the strength of trophic interactions are likely to induce autocorrelation in the noise, which will affect the estimated time to extinction (Halley and Kunin 1999, Heino et al. 2000). Thus, our estimates (Appendix) should be considered only as indicative for real extinction risks.

Our finding (Fig. 3) that larger populations are necessary to produce a given time to extinction at the fast end of the slow-fast continuum of life history variation (Sæther and Bakke 2000) rather than at the slow end seems to be in contrast to results from analyses of life history correlates of global extinction risks in birds (Bennett and Owens 1997, 2000), as well as in other vertebrate taxa (Reynolds 2003). These studies suggest that, in general, long-lived species that delay maturity face a larger risk of extinction than do highly reproductive species with a short life expectancy. One reason for this discrepancy may be that we have corrected for the confounding effect of population size. Accordingly, in an analysis of population lifetimes of mammals in North American national parks, population persistence time increased with initial population size (Newman 1995). After correcting for population size, longer survival times were found in species with delayed age at maturity, similar to the pattern (Fig. 3) recorded in our study.

An important tool in conservation of rare and endangered species has been the classification of species into the different vulnerability categories of the IUCN (2001). Although originally based on a quantitative approach (Mace and Lande 1991), the data necessary for such analyses are rarely available and the risk classification is then based on indirect evidence, mainly related to the overall abundance of the species in question. Even in well-studied taxa such as birds and mammals, data on temporal trends in population sizes of most rare and endangered species are lacking. Our results suggest that this approach will underestimate the risk of extinction because there will be a lag in time before extinction starts to occur as a consequence of a change in the population dynamics (Figs. 1 and 2). Thus, information on trends or population dynamics, and not only on population size, must be included when 
designing overall conservation strategies for threatened or vulnerable bird species.

Our analyses focus on the risk of extinction of local populations. Interpolating these results up to a larger geographical scale to improve of our understanding of processes affecting extinction on regional or global scales is difficult because such generalizations must involve strong assumptions about the pattern of interchange of individuals among local populations (Engen et al. 2002). Our results suggest that a general classification of interspecific variation in extinction risk of birds will be influenced by the relationship between total population size and the position of the species along the slow-fast continuum of life history variation (Sæther and Bakke 2000). For instance, in British birds, total population sizes as well as average abundance of a species were related to several life history characteristics (Blackburn et al. 1996): abundance decreased with age at maturity, life span, and lifetime reproduction. Thus, this suggests that, at a regional level, species with life history characteristics associated with a low risk of extinction (Fig. 3), in general, were less abundant than species located toward the fast end of the slow-fast life history continuum.

\section{ACKNOWLEDGMENTS}

The authors would like to express a sincere thanks to the help from J. Blondel, H. Christensen, J. De Laet, A. A. Dhondt, J.-D. Ludwigs, J. McMeeking, J. T. Nielsen, I. Owens, J. H. van Balen, and J. Visser. R. Freckleton helped with the phylogenetic analyses. R. Lande is thanked for critical comments on several drafts of the manuscript. Financial support was received from the European Commission (project METABIRD), the Research Council of Norway (Strategic University Program), Academy of Finland, and NSF (IBN9507365 and IBN-009702). This is publication number 3389 from NIOO-KNAW Netherlands Institute of Ecology.

\section{Literature Cited}

Beissinger, S. R., and D. R. McCullough. 2002. Population viability analysis. University of Chicago Press, Chicago, Illinois, USA.

Beissinger, S. R., and M. I. Westphal. 1998. On the use of demographic models of population viability in endangered species management. Journal of Wildlife Management 62: 821-841.

Belovsky, G. E., C. Mellison, C. Larson, and P. A. Van Zandt. 1999. Experimental studies of extinction dynamics. Science 286: 1175-1177.

Bennett, P. M., and I. P. F. Owens. 1997. Variation in extinction risk among birds: chance or evolutionary predisposition? Proceedings of the Royal Society of London Series B 263:401-408.

Bennett, P. M., and I. P. F. Owens. 2002. Evolutionary ecology of birds. Oxford University Press, Oxford, UK.

Blackburn, T. M., J. H. Lawton, and R. D. Gregory. 1996. Relationships between abundances and life histories of British birds. Journal of Animal Ecology 65:52-62.

Brooks, T. M., S. L. Pimm, and N. J. Collar. 1997. Deforestation predicts the number of threatened birds in insular southeast Asia. Conservation Biology 11:382-394.

Brooks, T. M., S. L. Pimm, V. Kapos, and C. Ravilious. 1999. Threat from deforestation to montane and lowland birds and mammals in insular South-East Asia. Journal of Animal Ecology 68:1061-1078.
Burgman, M. A., S. Ferson, and H. R. Akçakaya. 1993. Risk assessment in conservation biology. Chapman and Hall, London, UK.

Caughley, G. 1994. Directions in conservation biology. Journal of Animal Ecology 63:215-244.

Dennis, B., P. Munholland, and J. M. Scott. 1991. Estimation of growth and extinction parameters for endangered species. Ecological Monographs 61:115-143.

De Valpine, P., and A. Hastings. 2002. Fitting population models incorporating process noise and observation error. Ecological Monographs 72:57-76.

Drake, J. M., and D. M. Lodge. 2004. Effects of environmental variability on extinction and establishment. Ecology Letters 7:26-30.

Ellner, S. P., J. Fieberg, D. Ludwig, and C. Wilcox. 2002. Precision of population viability analysis. Conservation Biology 16:258-261.

Engen, S., Ø. Bakke, and A. Islam. 1998. Demographic and environmental stochasticity: concepts and definitions. Biometrics 54:840-846.

Engen, S., R. Lande, and B.-E. Sæther. 2002. The spatial scale of population fluctuations and quasi-extinction risk. American Naturalist 160:439-451.

Engen, S., R. Lande, and B.-E. Sæther. 2003. Demographic stochasticity and Allee effects in populations with two sexes. Ecology 84:2378-2386.

Fagan, W. F., E. Meir, J. Prendergast, A. Folarin, and P. Karieva. 2001. Characterizing population vulnerability for 758 species. Ecology Letters 4:132-138.

Ferraz, G., G. J. Russell, P. C. Stouffer, R. O. Bierregaard, Jr., S. L. Pimm, and T. E. Lovejoy. 2003. Rates of species loss from Amazonian forest fragments. Proceedings of the National Academy of Sciences (USA) 100:14069-14073.

Fieberg, J., and S. R. Ellner. 2000. When is it meaningful to estimate an extinction probability? Ecology 81:2040-2047.

Fisher, D. O., S. P. Blomberg, and I. P. F. Owens. 2003. Extrinsic versus intrinsic factors in the decline and extinction of Australian marsupials. Proceedings of the Royal Society of London Series B 270:1801-1808.

Freckleton, R. P., P. H. Harvey, and M. D. Pagel. 2002. Phylogenetic analysis and comparative data: a test and review of evidence. American Naturalist 160:712-726.

Groom, M., and M. A. Pascual. 1998. The analysis of population persistence: an outlook on the practice of viability analysis. Pages 4-27 in P. L. Fiedler and P. Kareiva, editors. Conservation biology. Second edition. Chapman and Hall, New York, New York, USA.

Halley, J. M., and V. E. Kunin. 1999. Extinction risk and the $1 / f$ family of noise models. Theoretical Population Biology 56:215-230.

Heino, M., J. Ripa, and V. Kaitala. 2000. Extinction risk under coloured environmental noise. Ecography 23:177184.

Henle, K., S. Sarre, and K. Wiegand. 2004. The role of density regulation in extinction processes and population viability analysis. Biodiversity and Conservation 13:9-52.

Houghton, J. T., Y. Ding, D. J. Griggs, M. Noguer, P. J. Van der Linden, and D. Xiasou. 2001. Climate change 2001. Cambridge University Press, Cambridge, UK.

Inchausti, P., and J. Halley. 2003. On the relation between temporal variability and persistence time in animal populations. Journal of Animal Ecology 72:899-908.

IUCN. 2001. IUCN Red List categories. Gland, Switzerland.

Jones, K. E., A. Purvis, and J. L. Gittleman. 2003. Biological correlates of extinction risks in bats. American Naturalist 161:601-614.

Karlin, S., and H. M. Taylor. 1981. A second course in stochastic processes. Academic Press, New York, New York, USA. 
Lande, R. 1993. Risks of population extinction from demographic and environmental stochasticity and random catastrophes. American Naturalist 142:911-927.

Lande, R. 1998. Demographic stochasticity and Allee effect on a scale with isotrophic noise. Oikos 83:353-358.

Lande, R., S. Engen, and B.-E. Sæther. 2003. Stochastic population dynamics in ecology and conservation. Oxford University Press, Oxford, UK.

Lande, R., S. Engen, B.-E. Sæther, F. Filli, E. Matthysen, and H. Weimerskirch. 2002. Estimating density dependence from population time series using demographic theory and life-history data. American Naturalist 159:321-337.

Leigh, E. G., Jr. 1981. The average lifetime of a population in a varying environment. Journal of Theoretical Biology 90:213-239.

Ludwig, D. 1996a. Uncertainty and the assessment of extinction probabilities. Ecological Applications 6:10671076.

Ludwig, D. 1996b. The distribution of population survival times. American Naturalist 147:506-526.

Ludwig, D. 1999. Is it meaningful to estimate a probability of extinction? Ecology 80:298-310.

Mace, G. M., and R. Lande. 1991. Assessing extinction threats: toward a reevaluation of IUCN threatened species categories. Conservation Biology 5:148-157.

May, R. M. 1981. Models for single populations. Pages 529 in R. M. May, editor. Theoretical ecology. Second edition. Blackwell Scientific Publications, Oxford, UK.

May, R. M., J. H. Lawton, and N. E. Stork. 1995. Assessing extinction rates. Pages 1-24 in J. H. Lawton and R. M. May, editors. Extinction rates. Oxford University Press, Oxford, UK.

Morris, W. F., and D. F. Doak. 2002. Quantitative conservation biology: theory and practice of population viability analysis. Sinauer Associates, Sunderland, Massachusetts, USA.

Newman, W. 1995. Extinction of mammal populations in western North American national parks. Conservation Biology 9:512-526.

Owens, I. P. F., and P. M. Bennett. 2000. Ecological basis of extinction risk in birds: habitat loss versus human persecution and introduced predators. Proceedings of the $\mathrm{Na}$ tional Academy of Sciences (USA) 97:12144-12148.

Pagel, M. D. 1999. Inferring the historical patterns of biological evolution. Nature 401:877-884.

Pimm, S. L. 1991. The balance of nature? University of Chicago Press, Chicago, Illinois, USA.

Pimm, S. L., and R. A. Askins. 1995. Forest losses predict bird extinctions in eastern North America. Proceedings of the National Academy of Sciences (USA) 92:9343-9347.

Pimm, S. L., J. Diamond, T. M. Reed, G. J. Russell, and J. Verner. 1993. Times to extinction for small populations of large birds. Proceedings of the National Academy of Sciences (USA) 90:10871-10875.
Pimm, S. L., H. L. Jones, and J. Diamond. 1988. On the risk of extinction. American Naturalist 132:757-785.

Pimm, S. L., G. J. Russell, J. L. Gittleman, and T. M. Brooks. 1995. The future of biodiversity. Science 269:347-350.

Purvis, A., J. L. Gittleman, G. Cowlishaw, and G. M. Mace. $2000 \mathrm{~b}$. Predicting extinction risk in declining species. Proceedings of the Royal Society of London Series B 267: 1947-1952.

Purvis, A., K. E. Jones, and G. M. Mace. 2000a. Extinction. BioEssays 22:1123-1133.

Reynolds, J. D. 2003. Life histories and extinction risk. Pages 195-217 in T. M. Blackburn and K. J. Gaston, editors. Macroecology. Blackwell Publishing, Oxford, UK.

Rosenzweig, M. L. 1995. Species diversity in space and time. Cambridge University Press, Cambridge, UK.

Sæther, B.-E., and Ø. Bakke. 2000. Avian life history variation and contribution of demographic traits to the population growth rate. Ecology 81:642-653.

Sæther, B.-E., and S. Engen. 2002. Pattern of variation in avian population growth rates. Philosophical Transactions of the Royal Society of London Series B 357:1185-1196.

Sæther, B.-E., and S. Engen. 2003. Routes to extinction. Pages 218-236 in T. Blackburn and K. Gaston, editors. Macroecology. Blackwell Publishing, Oxford, UK.

Sæther, B.-E., S. Engen, A. Islam, R. McCleery, and C. Perrins. 1998. Environmental stochasticity and extinction risk in a population of a small songbird, the great tit. American Naturalist 151:441-450.

Sæther, B.-E., S. Engen, R. Lande, P. Arcese, and J. N. M. Smith. 2000. Estimating time to extinction in an island population of song sparrows. Proceedings of the Royal Society of London Series B 267:621-626.

Sæther, B.-E., S. Engen, R. Lande, C. Both, and M. E. Visser. 2002a. Density dependence and stochastic variation in a newly established population of a small songbird. Oikos 99:331-337.

Sæther, B.-E., S. Engen, and E. Matthysen. 2002b. Demographic characteristics and population dynamical patterns of solitary birds. Science 292:2070-2073.

Sæther, B.-E., et al. In press. Life history variation predicts the effects of demographic stochasticity on avian population dynamics. American Naturalist.

Schoener, T. W., J. Clobert, S. Legendre, and D. A. Spiller. 2003. Life-history models of extinction: a test with island spiders. American Naturalist 162:558-573.

Schoener, T. W., and D. A. Spiller. 1992. Is extinction rate related to temporal variability in population size? An empirical answer for orb spiders. American Naturalist 139: 1176-1207.

Simberloff, D. 1992. Doe species-area curves predict extinction in fragmented forest? Pages 75-89 in T. C. Whitmore and J. A. Sayer, editors. Tropical deforestation and species extinction. Chapman and Hall, London, UK.

\section{APPENDIX}

A table showing the estimates of the population parameters is available in ESA's Electronic Data Archive: Ecological Archives E086-035-A1. 